\title{
Una aproximación desde la etnografía focalizada al papel de la religiosidad durante la vivencia del cáncer de mama'
}

\section{An approach from ethnography focused on the role of religiosity during the experience of breast cancer}

\section{Celmira Laza-Vásquez ${ }^{\mathrm{a}}$}

(iD) https://orcid.org/0000-0002-3264-5837

E-mail: celmira.lazaळudl.cat

\section{Montserrat Gea-Sánchez ${ }^{\mathrm{a}}$}

(iD) https://orcid.org/0000-0001-5143-3314

E-mail: montse.geaœudl.cat

anniversidad de Lleida. Facultad de Enfermería y Fisioterapia. Lleida, Espanha.

\author{
Correspondencia \\ Celmira Laza-Vasquez \\ Universidad de Lleida, Facultad de Enfermería y Fisioterapia. \\ Carrer Montserrat Roig, 2 CP: 25198, Lleida, España.
}

\section{Resumen}

En este trabajo se exploró el papel de la religiosidad durante la vivencia del cáncer de mama de un grupo de mujeres del sur de Colombia. Se realizó una etnografía focalizada donde participaron 17 mujeres que padecen cáncer de mama en cualquier estadio de la enfermedad y que habían concluido el primer año de tratamiento. La información se recolectó a través de un grupo de discusión y diez entrevistas semiestructuradas. El análisis temático se utilizó para el tratamiento de los datos. Para las mujeres, la religiosidad fue esencial para la aceptación del padecimiento, al asumirlo como una oportunidad de crecimiento personal y, además, la consideraron una fuente de afrontamiento durante el padecimiento. Se concluye que acercarse al papel de la religiosidad, desde las voces de las mujeres, devela el esencial papel que juega durante la trayectoria del cáncer de mama y la imperiosidad de la inclusión de la atención religiosa como un componente esencial para las mujeres durante el padecimiento del cáncer de mama. Asimismo, es un llamado a la importancia que la investigación sobre este padecimiento no se centre solo desde una perspectiva biomédica.

Palabras claves: Neoplasia de la Mama; Religiosidad; Acontecimientos que Cambian la Vida; Investigación Cualitativa.

1 Agradecimientos a la Universidad Surcolombiana y Universidad de Lleida por la financiación del estudio. 
This work explored the role of religiosity during the experience of breast cancer of a group of women in southern Colombia. A focused ethnography was carried out where 17 women who suffer from breast cancer at any stage of the disease and who had completed the first year of treatment participated. The information was collected through a discussion group and ten semistructured interviews. The thematic analysis was used to treat the data. For the women, religiosity was essential for the acceptance of the illness, assuming it as an opportunity for personal growth and also, they considered it a source of coping during the illness. It is concluded that approaching the role of religiosity, from the voices of women, reveals the essential role it plays during the breast cancer trajectory and the need of the inclusion of religious care as an essential component for women during the illness. It also calls for the importance that research on this illness not be focused only from a biomedical perspective.

Keywords: Breast Neoplasms; Religiosity; Life Change Events; Qualitative Research.
La presentación del cáncer de mama (CM) en Colombia continúa siendo desalentadora por el aumento sostenido en su presentación y la mortalidad femenina que ocasiona (Bray et al., 2018). Para el 2020, el CM siguió ocupando el primer lugar entre todos los cánceres en las mujeres, con una incidencia estimada de 59.9 y una mortalidad de 17 por cada 100 mil habitantes (GLOBOCAN, c2020).

Ser diagnosticada con CM puede ser una "experiencia traumática" para las mujeres al suponer un padecimiento que se asocia con la muerte, y generar una serie de respuestas emocionales como estrés, desesperación, incertidumbre, miedo, ansiedad, dolor, desesperanza y conmoción; lo que hace que las mujeres sean consideradas "emocionalmente vulnerables" (Liamputtong; Suwankhong, 2015).

La mayoría de las mujeres que padecen CM pueden verse rodeadas de mitos y connotaciones que van mucho más allá de la comprensión clínica objetiva de la enfermedad y enfrentan la incertidumbre del diagnóstico, del resultado del tratamiento y la sobrevivencia (Assaf; Holroyd; Lopez, 2017). Lo anterior se relaciona con el fatalismo religioso que explica el cáncer como una cuestión de destino predeterminado y/o retribución divina, lo que está inextricablemente vinculado a la religión (Liamputtong; Suwankhong, 2015).

La religión les permite a las personas asignar un significado a los eventos adversos de la viday, aunque es difícil de definir, es una necesidad, ayuda a encontrar un equilibrio de la vida y es un recurso para hacer frente a las crisis (Lynn; Yoo; Levine, 2014). La religiosidad es una expresión de la religión (Sheppard et al., 2018) y esta guía las creencias religiosas al ser un medio para que los individuos expresen su espiritualidad a través de la adopción de valores, creencias y prácticas rituales (asistencia a servicios religiosos, participación en actividades religiosas, fe intrínseca, creencias, actitudes y prácticas religiosas; e identificación y afiliación religiosa, entre otros) que dan respuesta a las principales preguntas sobre la vida y la muerte (Roh; Burnette; Lee, 2018). La religiosidad se asocia con principios fundamentales específicos que se organizan en torno a distintos sistemas de creencias, prácticas 
y rituales que tienen lugar dentro de una comunidad e involucran prácticas estructuradas, así como creencias teológicas (Hunter; Costas; Gany, 2015).

Estudios realizados en países desarrollados muestran que para las mujeres que padecen CM, la religiosidad juega un papel fundamental en el afrontamiento del diagnóstico, el tratamiento y la sobrevivencia (Sheppard et al., 2018). Sin embargo, también se ha relacionado con la disminución del bienestar y aumento de la depresión al sentir que el cáncer se debe a la ira o castigo de Dios; y porque puede convertirse en una barrera que retrasa el diagnóstico por la creencia de que solo Dios tiene el poder para curar (Gall; Bilodeau, 2020).

Ante la controversia sobre el papel que juega la religiosidad durante el CM y los escasos estudios recientes sobre el tema en Colombia, se exploró el papel de la religiosidad durante la vivencia del CM de un grupo de mujeres del sur de Colombia. Este estudio hace parte de uno de mayor alcance que exploró los itinerarios terapéuticos de las mujeres durante el padecimiento de la enfermedad.

\section{Métodos}

Se realizó una etnografía focalizada, diseño contemporáneo enfocado en los pequeños elementos de la sociedad y en las vidas de las personas que están "social y culturalmente fragmentadas y diferenciadas"; centrándose en el estudio de las experiencias compartidas de un fenómeno más limitado y predeterminado de pequeños grupos sociales para comprender problemas sociales específicos, la interrelación entre las personas y el contexto social en que viven y comparten los participantes, sus perspectivas sobre los eventos y cuestiones sociales (Rashid; Caine; Goez, 2015).

El estudio se realizó durante el año 2018 en la ciudad de Neiva, capital y municipio más importante del Departamento del Huila y de la región del sur de Colombia. Este Departamento ocupa el cuarto lugar en la incidencia del CM en el país, manteniendo una tendencia ascendente; siendo la incidencia en 2018 de 16 casos por cada 100 mil mujeres (SICC, 2018).

El escenario de la investigación fue la Liga Contra el Cáncer-Seccional Huila, organización sin ánimo de lucro en la región Surcolombiana que defiende los derechos de las personas que padecen cáncer y, a la vez, presta servicios de salud a todo el Departamento. El primer paso de la investigación consistió en identificar a las mujeres en los registros de los últimos 5 años de la organización y fueron contactadas e invitadas por una enfermera que labora en la misma, la cual actúo como portera de campo (Hammersley; Atkinson, 1994) para la entrada de las mujeres en el estudio.

A través de un muestreo conveniente se configuró una muestra con mujeres que padecen CM en cualquier estadio de la enfermedad y que habían superado el primer año de tratamiento. Se realizó un grupo de discusión inicial con 5 mujeres que duró 90 minutos y tuvo la finalidad de realizar una inmersión y exploración en el tema, y definir, junto con la revisión de la literatura publicada, los temas para las entrevistas semiestructuradas. Este guión incluyó la información biográfica de las mujeres, la experiencia de recibir un diagnóstico de CM y el papel que jugó la religión durante el trayecto de la enfermedad.

Las entrevistas tuvieron una duración entre 60 y 90 minutos y se realizaron hasta no encontrar nuevos hallazgos, con lo cual se consideró la saturación de la información. No se ofreció ningún incentivo financiero/material a las mujeres para participar, pero sí el pago del desplazamiento hasta el sitio de las entrevistas. El grupo de discusión y las entrevistas los dirigió la investigadora principal y fueron grabados en audio y transcritos íntegramente en las siguientes 48 horas.

Inicialmente, las transcripciones fueron leídas en varias ocasiones y se realizó una primera codificación de forma independiente por las dos investigadoras. Posteriormente, los códigos se compararon, fusionaron y discutieron para llegar a un consenso, y en casos de desacuerdos, se resolvieron después de varias rondas de análisis. Los códigos se organizaron en temas y subtemas en línea con el consenso alcanzado, que se revisaron, validaron y definieron siguiendo la propuesta de análisis temático de Clarke y Braun (2013). El análisis se hizo con el apoyo del programa Atlas.ti 8. Una vez elaborados los resultados, se presentaron a las participantes para su revisión y estos fueron aceptados sin sugerencias. 
Se tuvieron en cuenta los aspectos éticos para investigaciones con seres humanos: todas las participantes firmaron una forma de consentimiento informado individual, se garantizó la confidencialidad de los datos mediante la anonimización de las transcripciones y asignación de un número para identificar a las participantes. El protocolo del estudio fue aprobado por el Comité de Ética en Investigación de la Facultad de Salud de la Universidad Surcolombiana.

\section{Resultados}

La muestra se constituyó con 17 mujeres, con una edad promedio de 40 años (rango 27-52 años) y fueron diagnosticadas en promedio a los 364 años (rango de 25-50 años). Solo una era soltera y sin hijos, catorce de ellas se declararon católicas practicantes y las restantes, cristianas evangélicas. A excepción de tres, el resto pertenecían a un estrato socioeconómico bajo y solo siete contaban con estudios universitarios/superiores.

\section{Aceptar el padecimiento}

La religiosidad puede amortiguar el trauma psicológico de un diagnóstico de cáncer al permitirle a las personas conceptualizar la experiencia del padecimiento como parte de un plan más amplio establecido por un ser superior y, por lo tanto, centrarse en pensamientos positivos como la fe y la supervivencia, y disminuir los pensamientos negativos como la culpa y la muerte (Gesselman et al., 2017).

La religiosidad tiene una fuerte influencia en el bienestar subjetivo por lo que no debe subestimarse el impacto positivo en las vidas de las mujeres que padecen CM (Villani et al., 2019). Diferentes investigaciones muestran como la religiosidad ayuda en el ajuste durante una enfermedad potencialmente mortal, al compensar las pérdidas y mantener activamente sus vidas personales a través de un proceso de aprendizaje que les permite crear o descubrir nuevas oportunidades que podrían conducir a mantener u obtener algo de disfrute de la vida, incluso mientras el sufrimiento continúa durante el padecimiento y la recuperación (Zargani et al., 2018). Asimismo, les permite volverse optimistas frente a la enfermedad y reafirmar el propósito de luchar activamente contra ella; mejorar el comportamiento frente al estrés generado y desarrollar actitudes emocionales y psicológicas positivas que minimizan la angustia (Ahmadi et al., 2015).

Varios estudios muestran como la religiosidad y sus expresiones emergen con gran fuerza ante un diagnóstico de CM; al ser este un evento que genera una "interrupción biográfica" en todas las estructuras de la vida cotidiana de las mujeres (Liamputtong; Suwankhong, 2015). Coincidiendo con la bibliografía existente, para las participantes recibir el diagnóstico de $\mathrm{CM}$ fue una noticia abrumadora que las desestabilizó y alteró todos sus planes presentes y futuros; perdiendo importancia el resto de cosas y orientando todas sus fuerzas en luchar contra la enfermedad y sobrevivir a ella.

“[...] apenas recibí el diagnóstico me quedó sobrevivir y salir de esta. A mí no me importó el trabajo, la plata, las tareas, nada. O sea, a ti te entregan una patología y el mundo literalmente se detiene. [...] ¡Explotó una bomba atómica y tú quedaste de pie! ¿Qué hago? ¿Para dónde cojo?”. (GD Participante 4)

Por esto, no vieron otra opción para explicar tan duro diagnóstico que asumirlo como parte de un plan de Dios que tenía un propósito en sus vidas, por lo que debían aceptarlo. Así, le pidieron a Dios que las acompañaran en el trayecto que iban a iniciar al considerar que ellas solas no podrían hacerlo. Solo de la mano de Dios serían capaces de enfrentar la enfermedad y el duro tratamiento, y no tenían otra opción que "poner" sus vidas en sus manos para vencer la enfermedad.

"Sólo le dije: de rodillas, te supliqué que nunca me fuera a pasar esto, pero ahora que me está tocando, ¡Cógeme duro porque contigo lo puedo todo, pero sin ti no puedo nada! Y esa fue mi oración en el momento. Le dije: Dios mío cógeme porque siento que estoy cayendo en un hueco. Cógeme duro, me siento morir. Por eso yo le dije: no me sueltes de la mano, todo lo puedo contigo". (GD Participante 2)

Aceptar el CM como la voluntad divina no es difícil de comprender en una sociedad con fuertes valores religiosos como la colombiana (Laza et al., 2019), por 
lo que, para las participantes y para otras mujeres que han padecido CM, esta enfermedad se convirtió en parte de su destino, una expresión de sus luchas diarias contra la enfermedad y una prueba de fe que será recompensada en el más allá (Hammoudeh; Hogan; Giacaman, 2016). Así, la aceptación de la voluntad de Dios es una característica clara e importante de la forma en que las mujeres comprenden la aparición del CM y la aceptan sin debatirlo; lo que le otorga un significado al cáncer y se relaciona con cambios positivos percibidos en sus vidas (Carney; Park, 2018).

Por esto, las participantes decidieron no enfrentarse a la voluntad divina sino enfocarse en luchar contra la enfermedad, acompañadas de Dios.

“[...] yo no nunca renegué de tenerla [cáncer]. No, yo la asumí. [...] Yo la recibí ¿Sí? Con los brazos abiertos $y$ vamos a luchar, vamos a seguir, voy a salir de esta iQue sea lo que Dios quiera!". (Participante 13)

Solo dos de ellas, al recibir el diagnóstico, cuestionaron a Dios por haberles enviado esa enfermedad, aunque posteriormente reflexionaron sobre los designios divinos y los aceptaron con resignación cristiana.

"Antes de que se me quitara los alientos, peleaba era con Dios ¿Qué para que me había puesto la enfermedad? Cuando ya pasa el tiempo y uno reflexiona, dice: Dios sabe cómo hace sus cosas. Pues uno empieza a resignarse y decir: luchamos [silencio]". (Participante 16)

Para las participantes, como para otras mujeres que reciben un diagnóstico de $\mathrm{CM}$, solo les quedó confiar en Dios por ser Él la fuente de la curación de la enfermedad (Hammoudeh; Hogan; Giacaman, 2016). Como parte de la aceptación de los designios divinos y la dura prueba que Dios puso en sus vidas, consideraron que solo podrían sobrevivir al CM fortaleciéndose su fe en Él.

"[...] lo más importante es confiar en Dios y pedirle a Dios para que lo pueda ayudar a uno ¿Quién lo ayuda a uno si no Dios? Y no, gracias Dios yo contaba con buena suerte de que acá mi proceso [tratamiento] ha sido muy rápido". (Participante 11)
Sentir que iban de la mano de Dios y confiar en Él les proporcionó una sensación de control sobre esta difícil situación, y les generó consuelo: "Uno dice: pues, bueno si esto me pasó a mi es por algo y pues saberlo como llevar y confiar en Dios de que todo va a seguir saliendo bien" (Participante 12).

Así, la experiencia del CM es un desafío en las vidas de las mujeres que promueve el crecimiento personal; lo que ayuda a encontrar un propósito y un significado más amplio (todas las cosas suceden por una razón) a la enfermedad (Roh; Burnette; Lee, 2018).

Un hallazgo novedoso del estudio es el agradecimiento de las participantes por ser ellas las destinatarias del cáncer y que no fueran otros miembros de sus familias como sus hijos(as), esposos y padres ya que no hubieran podido resistir el dolor de verlos en una situación similar a la suya. Reflexionaron que al ser mujeres eran más fuertes, capaces de hacerle frente a la enfermedad y a la muerte; y de aceptar lo que les había "tocado" con cristiana resignación. Asimismo, a diferencia de sus hijos(as), ellas ya habían vivido suficiente.

" $Y$ después empecé a agradecer que me hubiera tocado a mí lo difícil, a mí la enfermedad y no que les hubiera tocado a mis hijos, a mi esposo porque más difícil. Es mejor y más fácil para uno aceptarse, asumir. Eso que vivir la enfermedad digamos, de un hijo o la muerte [silencio]!Uy no! ¡Dios nos libre! Pero que sea a un niño, a un hijo de uno ¡Ay, no terrible! Muy cruel". (Participante 6)

El testimonio anterior tiene de trasfondo el sacrificio personal como ideal femenino, y la idealización de la maternidad y de la madre sacrificada por sus hijos (Gilligan, 1982) como respuesta a una la ideología dominante de la "buena madre" como el ideal de las mujeres desinteresadas, sacrificadas y resilientes que dirigen sus esfuerzos a resolver los problemas y situaciones difíciles de sus hijos (Knight, 2013). También responde a la construcción social del CM en el cual impera un sistema androcéntrico y heterocentrado que, entre otras, demanda a las mujeres la preocupación por los demás durante la enfermedad (Sulik, 2007); priorizando las necesidades y deseos de los otros por encima de los propios por lo que, al asumir el rol de 
madre, pueden llegar al extremo de "perderse” a sí mismas por los demás (Howard; Bahr, 2001).

Por lo anterior, para las mujeres es importante dejar una huella de "género" al demostrar que, pese al CM, no se deja de ser mujer ni de ejercer los dos roles considerados socialmente como los principales: ser madre y esposa (Knight, 2013).

Igualmente, las participantes le agradecieron a Dios que su situación clínica, personal y familiar durante la enfermedad no fuera peor, en relación a otras mujeres diagnosticadas que conocían.

"Sí, era en una sola parte en el lado izquierdo [del seno]. Sí, tuve la bendición de Dios de que el mío [CM] estaba encapsulado, no tenía raíz [...] Entonces, ¿Cómo no voy a agradecer y a agachar la cabeza? Yo digo: creo que yo he sido bendecida”. (Participante 17)

"A otras que los maridos las dejan, [...] o se consiguen otra. [...] yo sentí que tenía todo para pasarla [CM] y que si no la pasaba era porque yo no quería [...]". (Participante 7)

\section{Una oportunidad de crecimiento personal $y$ reencuentro con Dios}

Altos niveles de religiosidad se han relacionado con experiencias positivas de crecimiento personal postraumático de mujeres sobrevivientes de CM (Gesselman et al., 2017). Así, al igual que los resultados de este estudio, padecer un CM es considerado por las mujeres como una oportunidad divina que se les brinda para su crecimiento y la transformación; para convertirse en mejores personas, más humildes, amables y apreciar a los otros (Devi; Fong, 2019).

La enfermedad es pensada como una oportunidad para reencontrarse con Dios, pedir perdón por sus pecados y realizar obras en favor de otros más necesitados; corregir sus errores, crecer y convertirse en mejores personas. Así, le encontraron significado al CM en sus vidas.

"Lo vi [CM] como la oportunidad en vida de reparar algunas cosas que de pronto no había hecho bien [...] Nunca lo vi como un castigo". (Participante 2)
"[...] yo ahorita trato de cómo, como llevar la fiesta en paz y no buscar pleitos. Yo digo: ¿Pero para que pelear? ¡Dios mío! [...] yo antes me estresaba por todo [...] yo era de una imposición: era lo que yo decía, lo que yo mandaba y lo que yo dijera. [...] hasta eso he mejorado [...]". (Participante 17)

Varias participantes consideraron que el padecimiento también se convirtió en una ocasión de crecimiento para los miembros de sus familias; al percibir que durante el trayecto de la enfermedad mejoró el ambiente familiar y sus familias se preocuparon más por ellas.

"[...] yo lloré tanto. jAy, lloré tanto, tanto! Y mire que, gracias a Dios, a raíz de todo esto, como que me han valorado más. O sea, me han dado un valor más dentro mi hogar. Antes yo les importaba ¿Sí? No puedo ser desagradecida con la vida [...] pero ahorita ellos son como más pendientes [...] No sé, ahora como que yo siento que la vida nos ha cambiado mucho, ya no peleamos. [...] ya no era como antes: discusiones, peleas, maltratos. Gracias a Dios ya no. Ahorita, gracias a Dios ya no se ve eso. Entonces pienso que ha servido por eso, porque me han valorado más”. (Participante 17)

Así, las participantes sintieron que enfrentarse al CM las había convertido en personas más fuertes, lo que las ayudó a sobrellevar la enfermedad: "[...] todo eso que viví, que pasé me hizo más fuerte mucho iMucho más fuerte! Sobre todo, siempre de la mano de Dios" (GD Participante 4).

Por esto, ofrecieron a Dios el sufrimiento que significó para ellas y sus familias la trayectoria de la enfermedad y el tratamiento.

"[...] cuando me pusieron esas quimios rojas [silencio] ¡No me quiero ni acordar! Yo vomitaba sentada en el baño y se me venía a la cabeza la imagen de Dios, cuando Él esta cogido como de un tronco. Yo le decía: Señor, tu sufriste mucho por la salvación del mundo, te ofrezco este sufrimiento, le ofrecía por mi mamá, mi papá que ya había fallecido, mi familia [...]”. (Participante 2) 


\section{Fuente de afrontamiento durante el padecimiento}

Las participantes consideraron que, si CM era la voluntad divina, Dios no las abandonaría y les daría la fortaleza para afrontarlo. Así lo expresó una de ellas: [...] lo que me ha dado mucha fortaleza, demasiada fortaleza, estar pegadita de la manito de Dios. (Participante 15)

Por esto, le pidieron que las acompañara durante el tratamiento y se aferraron a Él durante este duro proceso; sintiendo que Dios respondió a sus plegarias al lograr sortear los tratamientos sin grandes dificultades.

“[...] y me decían que le da vómito [quimioterapia]. Cosas así. Pero no, a mí gracias a Dios muy bendecida, no me dio nada”. (Participante 13)

"[...] a mí Dios se me manifestó antes de yo entrar a cirugía, yo tenía mucho miedo [...] y, en nombre de Dios, no siento dolor. Cuando yo desperté, también me decían: ¿Quieres morfina? Y yo: no, no quiero morfina [...]”. (GD Participante 4)

Además de brindarles fortaleza, las mujeres describieron cómo Dios les proporcionó los recursos para enfrentar la enfermedad, facilitándoles la gestión con sus aseguradoras lo que les evitó las barreras de acceso al tratamiento como la negación o demora en las autorizaciones de procedimientos, consultas con los especialistas, medicamentos y exámenes diagnósticos, entre otros.

"Yle dije: ¡Dios mío ayúdame y ábreme las puertas de la EPS [aseguradoras]que es lo más verraco [difícil] de abrir! Ábreme las puertas de las EPS, que todos mis trámites sean rápidos, que se vea tú mano [...]. Dios por favor ;Y asífue! [...] el 8 de marzo me entregan mi patología y el 13 de abril ya había pasado por médico general, ginecólogo, internista, biopsia, resultado de biopsia [...]. Yo ese día dije: ¡Ahí ya se vio la mano de Dios!" (GD Participante 12)

Como recursos, también les "envió" a diferentes personas que les ayudaron en las aseguradoras y en las instituciones hospitalarias donde las atendieron para adelantar el tratamiento sin obstáculos y solucionar los que se les presentaran.

"[...] gracias a mi Dios, como que le va, le va poniendo también a uno las cosas para solucionar y que las cosas se den. Ahí en Saludcoop [institución hospitalaria] hubo una muchacha [trabajadora] [...] y me colaboró mucho para que me agilizaran las citas con el especialista y todo. Como que mi Diosito me la puso ahí [...]. Me dijo: la voy a ayudar. Entonces ella me dijo: mire, tranquila que cuando le salgan los resultados de la biopsia usted viene y yo con mucho gusto le ayudo en cualquier cosa que usted necesite". (Participante 14)

Otro recurso divino fueron los médicos que Dios puso en su camino e hizo más llevable el tratamiento: "[...] yo le debo esto [sobrevivir] primero a Dios y después a los médicos ;Gracias a Dios caí en buenas manos!" (Participante 8).

Lo relatado por las mujeres participantes responde al concepto de afrontamiento religioso positivo, que no es más que una expresión de una relación segura con un Dios/poder superior solidario, contrario a uno distante y castigador; mediante el uso de técnicas cognitivas y conductuales para hacer frente a eventos estresantes de la vida ( $\mathrm{Ng}$ et al., 2017). El desarrollo regular de estrategias de afrontamiento religioso positivo entre las mujeres que padecen CM se ha descrito como un aspecto que genera un impacto positivo en el bienestar general, la adaptación y aceptación de la enfermedad; y proporciona consuelo emocional y esperanza (Mehrabi et al., 2015).

En este sentido, para las mujeres del estudio, la religiosidad jugó un papel fundamental para el afrontamiento positivo de la enfermedad; contrario a lo descrito en otras investigaciones donde se convirtió en un obstáculo para el bienestar de las mujeres y una barrera que interfiere con el adecuado desarrollo del tratamiento médico (Gall; Bilodeau, 2020).

\section{Prácticas religiosas: estrategias para acercarse más a Dios}

Una consecuencia de la influencia de la religiosidad en la trayectoria de la enfermedad 
de las participantes fue el desarrollo regular de varias prácticas. Esto se explica porque durante un padecimiento potencialmente mortal, las prácticas religiosas son efectivas en la regulación de las emociones, la inhibición del comportamiento y el autocontrol; disminuyendo el pensamiento pesimista y la desesperanza (Holt et al., 2012). Asimismo, reduce la angustia psicológica al aumentar la autoestima o las perspectivas constructivas de atribución que ayudan a encontrar sentido y significado a un evento traumático, mejorando la capacidad de afrontamiento (Fradelos et al., 2018).

A pesar de que todas las mujeres del estudio se declararon creyentes y que profesaban una religión (católica o cristiana evangélica), explicaron que antes del diagnóstico de CM se encontraban alejadas de sus iglesias y de las prácticas religiosas.

"Yo ya venía en la iglesia, yo ya había conocido la Misión carismática mucho más antes, pero cosas de la vida me habían alejado. Me alejé. Llegó esto [CM], entonces yo digo: no, [...] buscar ese apoyo espiritual, para uno es muy bueno". (Participante 15)

Sin embargo, al irrumpir el cáncer en sus vidas decidieron que debían acercarse más a Dios para fortalecer su fe, y contar con su apoyo y acompañamiento durante la enfermedad. Por esto, retomaron de forma regular varias prácticas religiosas como la oración, asistencia a servicios religiosos, la lectura de libros religiosos, entre otros, para pedirle a Dios su acompañamiento y que las hiciera fuertes para vencer la enfermedad.

"Pues la verdad, no era de las que me lo pase allá, en la iglesia cada 8 días. Yo soy muy católica, yo oro mucho, yo le pido mucho a mi Dios. Yo en ese momento [diagnóstico] le pedímucho a Él, que me diera mucho valor, mucha fortaleza [...] le pedía mucho a mi Diosito que me ayudara a salir adelante". (Participante 14)

El acompañamiento de sus líderes religiosos fue fundamental ya que las orientó en el desarrollo de sus prácticas religiosas.

“[...] la líder mía, en el proceso mío, estuvo muy pendiente. Ella todos los días me manda un devocional, todos los días me mandan audios. Hay eventos en la iglesia y cosas, y me dice: doña, me gustaría que fuera. Todos los días le manda una reflexión y todo eso como que le ayuda a uno [...]". (Participante 17)

También le hicieron promesas a Dios y participaron más asiduamente en sus comunidades religiosas.

"Yo me volví muy devota del Señor de la Divina Misericordia y le prometí que iba a ir cuando saliera de todo". (Participante 7)

"[...] hay unos muy buenos grupos de oraciones y los encontré [...]. Pero cuando ya llegué ahí, que ya me tocó a mí un tropiezo difícil, encontré personas que también han vivido muchas cosas diferentes y difíciles". (Participante 6)

En sintonía con varios estudios, las prácticas religiosas fueron esenciales para que las mujeres continuaran con sus vidas y desviaran la atención de las experiencias emocionales negativas (Roh; Burnette; Lee, 2018); sintieran una disminución de la carga del cáncer, de no encontrarse solas y de apoyo para sobrellevar los tratamientos y la recuperación (Assaf; Holroyd; Lopez, 2017).

Al finalizar el primer año de tratamiento, las prácticas religiosas fueron el medio para agradecer a Dios por permitirles "sobrevivir" a la enfermedad, a diferencia de otras mujeres conocidas en su misma situación y que habían fallecido. Así, se consideraron bendecidas, un milagro de Dios y un testimonio de su existencia. Así lo expresó una de ellas: "Yo decía: ¡Dios mío! Estar con vida, ganarle la batalla a esto es, mejor dicho [llanto]. Todas no tenemos esa bendición [...] nosotras somos un testimonio de Dios. Yno, pues por eso le digo: ¡Dios es tan perfecto!' (GD Participante 4)

\section{Consideraciones finales}

Acercarse a la influencia de la religiosidad, desde las voces de las mujeres, devela que este es un aspecto crucial que atraviesa la trayectoria durante la experiencia del CM; jugando un papel fundamental en la aceptación de la enfermedad, para asumir el padecimiento como una oportunidad de 
crecimiento personal, y una fuente de afrontamiento de la enfermedad.

A pesar de las limitaciones de los resultados, como circunscribirse al contexto de una región colombiana y la ausencia de la inclusión en el estudio de otros actores como los familiares más cercanos y los profesionales de la salud, estos hacen un llamado a los profesionales de la salud sobre la urgencia de incluir la atención religiosa en la atención y cuidado de las mujeres. La atención religiosa reconoce la religiosidad y espiritualidad de los pacientes, y propone atender estas necesidades en el entorno clínico; implicando el apoyo individual en tiempos de crisis para una búsqueda personal del significado de la vida (Balboni et al., 2010).

Por lo anterior, debe pensarse como un componente esencial en la atención y el cuidado de las mujeres durante el padecimiento; y es imprescindible que los profesionales se familiaricen, presten atención y evalúen el papel de la religiosidad para comprender y atender mejor las preocupaciones religiosas. Asimismo, para pensar en el desarrollo de intervenciones de cuidado espiritual.

La investigación futura debe continuar indagando y profundizando en el fenómeno de la religiosidad durante la vivencia del CM y, en especial, en el afrontamiento religioso, la influencia de la religiosidad en las actitudes y comportamientos durante la enfermedad, la atención religiosa y la sensibilidad de las(os) enfermeras(os) a la religiosidad de las mujeres.

Finalmente, estos resultados se suman a muchos otros que reclaman que la investigación sobre CM no se centre solo desde una perspectiva biomédica, sino también desde una sociocultural que considere cómo los factores de género, sociales, culturales y económicos impactan las experiencias de la enfermedad.

\section{Referencias}

AHMADI, Z.; DARABZADEH, F.; NASIRI, M. et al. The effects of spirituality and religiosity on wellbeing of people with cancer: a literature review on current evidences. Jundishapur Journal of Chronic Disease Care, Ahvaz, v. 4, n. 2, e28386, 2015. DOI: $10.1002 /$ cncr.29353
ASSAF, G. N.; HOLROYD, E.; LOPEZ, V. Isolation and prayer as means of solace for Arab women with breast cancer: an in-depth interview study. PsychoOncology, Hoboken, v. 26, n. 11, p. 1888-1893, 2017. DOI: $10.1002 /$ pon. 4402

BALBONI, T. A.; PAULK, M. E.; BALBONI, M. J. et al. Provision of spiritual care to patients with advanced cancer: associations with medical care and quality of life near death. Journal of Clinical Oncology, Alexandria, v. 28, n. 3, p. 445-452, 2010. DOI: 10.1200/ JC0.2009.24.8005

BRAY, F.; FERLAY, J.; SOERJOMATARAM, I. et al. Global cancer statistics 2018: GLOBOCAN estimates of incidence and mortality worldwide for 36 cancers in 185 countries. A Cancer Journal for Clinicians, Hoboken, v. 68, n. 6, p. 394-424, 2018. DOI: 10.3322/caac.21492

CARNEY, L. M.; PARK, C. L. Cancer survivors' understanding of the cause and cure of their illness: Religious and secular appraisals. PsychoOncology, Hoboken, v. 27, n. 6, p. 1553-1558, 2018. DOI: 10.1002/pon.4691

CLARKE, V.; BRAUN, V. Teaching thematic analysis: overcoming challenges and developing strategies for effective learning. The Psychologist, Leicester, v. 26, p. 120-123, 2013.

DEVI, M. K.; FONG, K. C. K. Spiritual experiences of women with breast cancer in Singapore: a qualitative study. Asia-Pacific Journal of Oncology Nursing, Alphen aan den Rijn, v. 6, n. 2, p. 145-150, 2019. DOI: 10.4103/apjon.apjon_77_18

FRADELOS, E. C.; LATSOU, D.; MITSI, D. et al. Assessment of the relation between religiosity, mental health, and psychological resilience in breast cancer patients. Contemporary Oncology, Poznan, v. 22, n. 3, p. 172-177, 2018. DOI: 10.5114/ wo.2018.78947

GALL, T. L.; BILODEAU, C. Attachment to God and coping with the diagnosis and treatment of breast cancer: a longitudinal study. Supportive Care in Cancer, Berlin, v. 28, n. 6, p. 2779-2788, 2020. DOI: $10.1007 /$ soo520-019-05149-6

GESSELMAN, A. N.; BIGATTI, S. M.; GARCIA, J. R. et al. Spirituality, emotional distress, 
and post-traumatic growth in breast cancer survivors and their partners: an actor-partner interdependence modeling approach. PsychoOncology, Hoboken, v. 26, n. 10, p. 1691-1699, 2017. DOI: $10.1002 /$ pon.4192

GILLIGAN, C. In a different voice: psychological theory and women's development. Cambridge: Harvard University Press, 1982.

GLOBOCAN GLOBAL CANCER OBSERVATORY. Estimated number of new cases in 2020, worldwide, females, all ages, c2020. Disponível em: <https://bit. ly/3iFZkzK>. Acesso em: 1 fev. 2021.

HAMMERSLEY, M.; ATKINSON, P. Etnografía: métodos de investigación. Barcelona: Paidos, 1994. HAMMOUDEH, W.; HOGAN, D.; GIACAMAN, R. From a death sentence to a disrupted life: Palestinian women's experiences and coping with breast cancer. Qualitative Health Research, Thousand Oaks, v. 27, n. 4, p. 487-496, 2016. DOI: $10.1177 / 1049732316628833$

HOLT, C. L.; SCHULZ, E.; CAPLAN, L. et al. Assessing the role of spirituality in coping among African Americans diagnosed with cancer. Journal of Religion and Health, Berlin, v. 51, n. 2, p. 507-521, 2012. DOI: $10.1007 / \mathrm{s10943-011-9453-0}$

HOWARD, M. B.; BAHR, K. S. Families and selfsacrifice: alternative models and meanings for family theory. Social Forces, Oxford, v. 79, n. 4, p. 1231-1258, 2001. DOI: 10.1353/sof.2001.0030 HUNTER, M.; COSTAS, R.; GANY, F. Missed opportunity: spirituality as a bridge to resilience in Latinos with cancer. Journal of Religion and Health, Berlin, v. 54, n. 6, p. 2367-2375, 2015. DOI: 10.1007/s10943-015-0020-y

KNIGHT, K. The changing face of the 'good mother': trends in research into families with a child with intellectual disability, and some concerns. Disability \& Society, Abingdonon-Thames, v. 28, n. 5, p. 66o-673, 2013. DOI: $10.1080 / 09687599.2012 .732540$

LAZA, C.; CERQUERA, E. Y.; FERRER, T. A. et al. El aborto legal en Colombia: opiniones de un grupo de estudiantes de Enfermería. Revista Facultad Nacional de Salud Pública, Medellín, v. 37, n. 1, p. 78-86, 2019. DOI: 10.17533/udea.rfnsp. v37n1a12

LIAMPUTTONG, P.; SUWANKHONG, D. Breast cancer diagnosis: biographical disruption, emotional experiences, and strategic management in Thai women with breast cancer. Sociology of Health \& Illness, Hoboken, v. 37, n. 7, p. 1086-1101, 2015. DOI: 10.1111/1467-9566.12282

LYNN, B.; YOO, G. J.; LEVINE, E. G. Trust in the Lord: religious and spiritual practices of African American breast cancer survivors. Journal of Religion and Health, Berlin, v. 53, n. 6, p. 1706-1716, 2014. DOI: 10.1007/s10943-013-9750-X

MEHRABI, E.; HAJIAN, S.; SIMBAR, M. et al. Coping response following a diagnosis of breast cancer: a systematic review. Electronic Physician, Mashhad, v. 7, n. 8, p. 1575-1583, 2015. DOI: 10.19082/1575

NG, G. C.; MOHAMED, S.; SULAIMAN, A. H. et al. Anxiety and depression in cancer patients: the association with religiosity and religious coping. Journal of Religion and Health, Edmonton, v. 56, n. 2, p. 575-59o, 2017. DOI: $10.1177 / 1609406915621421$

RASHID, M.; CAINE, V.; GOEZ, H. The encounters and challenges of ethnography as a methodology in health research. International Journal of Qualitative Methods, Thousand Oaks, v. 14, n. 5, p. 1609406915621421, 2015. DOI: $10.1177 / 1609406915621421$

ROH, S.; BURNETTE, C. E.; LEE, Y. S. Prayer and faith: spiritual coping among American Indian women cancer survivors. Health \& Social Work, Oxford, v. 43, n. 3, p. 185-192, 2018. DOI: 10.1093/ hsw/hlyo15

SHEPPARD, V. B.; WALKER, R.; PHILLIPS, W. et al. Spirituality in AfricanAmerican breast cancer patients: implications for clinical and psychosocial care. Journal of Religion and Health, Berlin, v. 57, n. 5, p. 1918-1930, 2018. DOI: 10.1007/s10943-018-0611-5

SICC SISTEMA DE INFORMACIÓN DE CÁNCER EN COLOMBIA. Mortalidad, 2018. Disponível em: <https://bit.ly/3vn5rf9>. Acesso em: 1 fev. 2021. 
SULIK, G. A. On the receiving end: women, caring, and breast cancer. Qualitative Sociology, Berlin, v. 3o, n. 3, p. 297-314, 2007. DOI: 10.1007/s11133007-9057-X

VILLANI, D.; SORGENTE, A.; IANNELLO, P. et al. The role of spirituality and religiosity in subjective well-being of individuals with different religious status. Frontiers in Psychology,
Lausanne, v. 10, p. 1525-1525, 2019. DOI : 10.3389/ fpsyg.2019.01525

ZARGANI, A.; NASIRI, M.; HEKMAT, K. et al. A survey on the relationship between religiosity and quality of life in patients with breast cancer: a study in Iranian Muslims. Asia-Pacific Journal of Oncology Nursing, Alphen aan den Rijn, v. 5, n. 2, p. 217-222, 2018. DOI: 10.4103/apjon.apjon_65_17

\section{Agradecimientos}

Las autoras desean expresar sus agradecimientos a las mujeres que participaron en el estudio, y a la Liga Contra el Cáncer-Seccional Huila y al grupo de investigación "Salud y grupos vulnerables" de la Universidad Surcolombiana por el apoyo para el desarrollo de la investigación.

\section{Contribución de los autores}

Laza-Vásquez: concepción, recolección de datos y análisis de datos, elaboración del manuscrito, redacción, discusión de resultados. Géa-Sánchez: concepción, análisis de datos, elaboración del manuscrito, redacción, discusión de resultados.

Recibido: 27/05/2021

Re-presentado: 08/02/2021; 27/05/2021

Aprobado: 02/06/2021 